\title{
Capacité d'ingestion des génisses : influence de la race, de l'âge et de la qualité du foin
}

\author{
P D'Hour 1, JB Coulon 2, JP Garel 1 \\ avec la collaboration technique de $A$ Mante et $P$ Maronne \\ 1 INRA, domaine de la Borie, 15190 Marcenat; \\ 2 INRA-CRVZ, lactation et élevage des ruminants, Theix 63122 Ceyrat; France
}

(Reçu le 7 décembre 1990; accepté le 28 juin 1991)

\begin{abstract}
Résumé - Les capacités d'ingestion de génisses de différentes races laitières spécialisées et rustiques (Pie Noire croisée Holstein, Montbéliarde, Tarine et Salers) ont été comparées 2 années consécutives lorsqu'elles étaient âgées de 1 et 2 ans en utilisant chaque année 2 foins de valeurs différentes. L'influence du mode de stabulation (libre et entravée) a également été mesurée. La capacité d'ingestion dépend essentiellement du poids vif qui représente $58 \%$ de la variance observée pour chaque classe d'ảge. Toutes classes d'âge confondues, la capacité d'ingestion augmente proportionnellement au poids vif élevé à la puissance 0,8 . A même poids vif, les animaux des différents types laitiers ont des capacités d'ingestion identiques, mais supérieures de $15 \%$ à celle des génisses Salers. Aucune interaction significative sur les quantités ingérées n'a été observée entre la race des génisses et la qualité du foin. En stabulation libre, les génisses augmentent leur capacité d'ingestion de $10 \%$ en moyenne par rapport à la stabulation entravée. Ces résultats correspondent à ceux de la bibliographie et sont en accord avec les méthodes utilisées en France pour prévoir les quantités de fourrages ingérées (système des Unités d'Encombrement).
\end{abstract}

génisse / race bovine / ingestion / foin

Summary - Voluntary feed intake in helfers: influence of breed, age and the hay characteristics. Voluntary feed intake was compared in heifers of different breeds: Holstein Friesian (dairy breed), Montbeliarde (dairy breed), Tarentaise (local dairy breed) and Salers (hardy breed). The animals were 1 or 2 yr old, except the Salers heifers which were only 1 yr old (table 1); they were divided into 2 groups which were fed at libitum with hay of different qualities (table II). Organic dry matter digestibilities ranged between 48-62\%. The heifers were housed in individual stalls, but some Holstein Friesian, Tarentaise and Salers heifers were also stabled in covered pens. Within breed, the variation in voluntary feed intake related to the body weight fell to $\approx 77 \%$. Within breed and within age the proportion was $58 \%$ (fig 1). In the 3 dairy breeds, feed intake increased with body weight raised to a power of 0.8 (fig 2). At the same body weight, the 3 dairy breeds had the same feed intake capacity (table III). Salers heifer intake was $\approx 15 \%$ lower than that of the dairy breed. The quality of hay did not interact with breed for feed intake. In open housing, heifers ingested $\approx 10 \%$ more than in individual stalls (table IV).

heifer / breed / intake / hay

* Étude réalisée dans le cadre du programme de Recherche-Développement des Alpes du Nord. 


\section{INTRODUCTION}

La mesure de la capacité d'ingestion des génisses a déjà fait l'objet de nombreux travaux. En France, ces travaux ont concerné essentiellement la génisse Pie Noire, choisie comme animal de référence pour le calcul des unités d'encombrement bovin (UEB) (Dulphy et al, 1987), et celles de races allaitantes (Agabriel et al, 1987a, 1987b). À même poids vif, la capacité d'ingestion des génisses de races laitières (Pie Noire, Normande et Montbéliarde) est identique mais différente de celle des races allaitantes (Troccon et al, 1988). D'autres travaux ont également montré des différences importantes de capacité d'ingestion entre races laitières et à viande (Taylor et al, 1986), ou même à l'intérieur de génotypes laitiers (Oldenbroek et Van Eldik, 1980).

Les objectifs de cette étude étaient essentiellement de préciser les différences de capacité d'ingestion de génisses de races laitières plus ou moins spécialisées et allaitantes, de formats adultes différents, et de déterminer l'influence de la qualité du foin sur ces différences. Par ailleurs, on a aussi étudié l'influence du mode de stabulation, libre ou entravée, sur les quantités ingérées par les génisses de 1 et 2 ans.

\section{CONDITIONS EXPÉRIMENTALES}

Pendant 2 hivers successifs, les quantités de foin ingérées par des génisses de 1 et 2 ans ont été mesurées. Cette étude a été réalisée sur le domaine INRA de Marcenat (Cantal, $1100 \mathrm{~m}$ d'altitude).

\section{Génisses}

Les génisses utilisées dans cet essai étaient de race Pie Noire $(P n-1 / 4$ de sang Frison et $3 / 4$
Holstein), Montbéliarde (Mo), Tarine (Ta) et Salers (Sa) (tableau I). Les génisses Salers n'ont participé à l'essai que le premier hiver, étant alors âgées de 11 mois. Les génisses laitières étaient, en moyenne, àgées de 13 mois ( $1^{\text {er }}$ hiver) et de 25 mois ( $2^{\theta}$ hiver). Les poids vifs au début de l'essai et les croissances en période expérimentale ont varié respectivement de 262 (Ta) à $316 \mathrm{~kg}$ (Sa) et de 330 (Sa) à $675 \mathrm{~g} / \mathrm{j}$ (Pn), la première année. Au début du second hiver, les génisses pesaient de 382 (Ta) à 449 kg $(P n)$, et les croissances en période expérimentale ont varié de 410 (Ta) à $485 \mathrm{~g} / \mathrm{j}$ (Pn : tableau I).

De la naissance à 30 mois, les génisses laitières ont été soumises à la même conduite d'élevage (allaitement artificiel et sevrage à 10 semaines). Les génisses Salers ont été allaitées jusqu'à 9 mois et sevrées au moment de la rentrée à l'étable. Leurs croissances $(780 \mathrm{~g} / \mathrm{j})$ pendant cette phase d'allaitement ont eté supérieures de $200 \mathrm{~g} / \mathrm{j}$ de celles des génisses laitières.

\section{Conduite et alimentation}

Au cours des 2 hivers, les mesures ont été réalisées, soit individuellement sur les génisses conduites en étable entravée (respectivement 59 et 47 les hivers 1 et 2), soit sur des groupes de 7 ou 8 génisses élevées en stabulation libre à logettes et non paillée (respectivement 22 et 15). Dès la rentrée à l'étable (7 novembre 1987 et 3 novembre 1988), les génisses ont été réparties entre les 2 types de stabulation et ont reçu le même foin à volonté pendant 3 semaines. A la fin de cette période, les génisses logées en stabulation entravée ont été réparties intrarace en 2 lots sur la base de leur poids et des quantités ingérées pendant cette première période. Chacun de ces 2 lots a alors recu un foin de qualité différente pendant 8 semaines (tableau II). Le foin, distribué aux génisses de la stabulation libre, était l'un de ces 2 foins (première année : foin 1, seconde année : foin 2).

Ces foins, de premier cycle, ont tous été récoltés au cours du mois de juillet à une altitude de $1100 \mathrm{~m}$, sur des prairies naturelles (PN) pour 3 d'entre eux et de dactyle (Da) pour le dernier. Chaque année, les meilleurs foins (foins $n^{\circ} 1$ ) ont été déprimés 40-50 j avant la fenai- 
Tableau I. Caractéristiques des animaux utilisés.

\begin{tabular}{lcccc}
\hline & Effectif & $\begin{array}{c}\text { Age } \\
\text { (jours) }\end{array}$ & $\begin{array}{c}\text { Poids vif (1) } \\
(\mathrm{kg})\end{array}$ & $\begin{array}{c}\text { Croissance (1) } \\
(\mathrm{g} / \mathrm{j})\end{array}$ \\
\hline Génisses « 1 an» & & & & \\
Pie-Noire & & & & \\
Montbéliarde & & 399 & 312 & 675 \\
& 15 & $( \pm 13)$ & $( \pm 21)$ & $( \pm 80)$ \\
Tarine & 382 & 288 & 630 \\
& 23 & $( \pm 22)$ & $( \pm 26)$ & $( \pm 113)$ \\
Salers & 397 & 262 & 500 \\
& 21 & $( \pm 29)$ & $( \pm 25)$ & $( \pm 90)$ \\
& & 347 & 316 & 325 \\
Génisses «2 ans" & $( \pm 7)$ & $( \pm 17)$ & $( \pm 130)$ \\
Pie-Noire & & & & \\
Montbéliarde & & & & \\
Tarine & & & & \\
& 22 & 767 & 487 & 485 \\
& 16 & $( \pm 13)$ & $( \pm 33)$ & $( \pm 180)$ \\
& & 744 & 452 & 450 \\
& & $( \pm 25)$ & $( \pm 28)$ & $( \pm 155)$ \\
& & 759 & 414 & 410 \\
& & $( \pm 32)$ & $( \pm 35)$ & $( \pm 145)$ \\
\hline
\end{tabular}

(1) Période expérimentale

son. Les foins de moins bonne qualité (foins $n^{\circ}$ 2) ont reçu de la pluie (3-5 j) au cours du séchage.

Les foins étaient distribués à volonté (8$10 \%$ de refus), 2 fois par jour avec un complément de tourteau de soja "48" qui représentait 4 à $6 \%$ de la matière sèche du foin ingéré et de $100 \mathrm{~g} / \mathrm{j}$ d'un composé minéral vitaminé $(10 \%$ de Phosphore-14\% de Calcium). De l'eau et du sel étaient disponibles à volonté.

\section{Mesures}

Les quantités de foin offertes ont été pesées tous les jours et les quantités refusées 6 jours par semaine. La teneur en matière sèche (MS) des aliments offerts et refusés a été mesurée 2 fois par semaine sur les foins et le tourteau. Un échantillon moyen a été conservé par aliment, pour déterminer les teneurs en matière organique (MO), cellulose brute (CB) et matières azotées totales (MAT). La valeur nutritive de chaque fourrage (tableau II) a été estimée à partir de la digestibilité in vivo de la matière organique (Andrieu et Demarquilly, 1987). Celle-ci a été obtenue, pour chaque fourrage, en utilisant des lots de 6 moutons pendant 2 périodes de mesures de $7 \mathrm{j}$ chacune et après une période d'adaptation de $14 \mathrm{j}$. La valeur d'encombrement (UEB) de chaque foin a été estimée à partir de la moyenne des quantités ingérées par les génisses Pie Noires, considérées comme l'animal de référence (Dulphy et al, 1987) et de la capacité d'ingestion théorique, compte tenu du poids, de ces mêmes génisses (Troccon et al, 1988).

Les génisses étaient pesées tous les $14 \mathrm{j}$ au cours de l'essai, et $2 \mathrm{j}$ successifs avant et après la période de mesure. Les croissances ont été 
Tableau II. Composition chimique et valeur nutritive des foins.

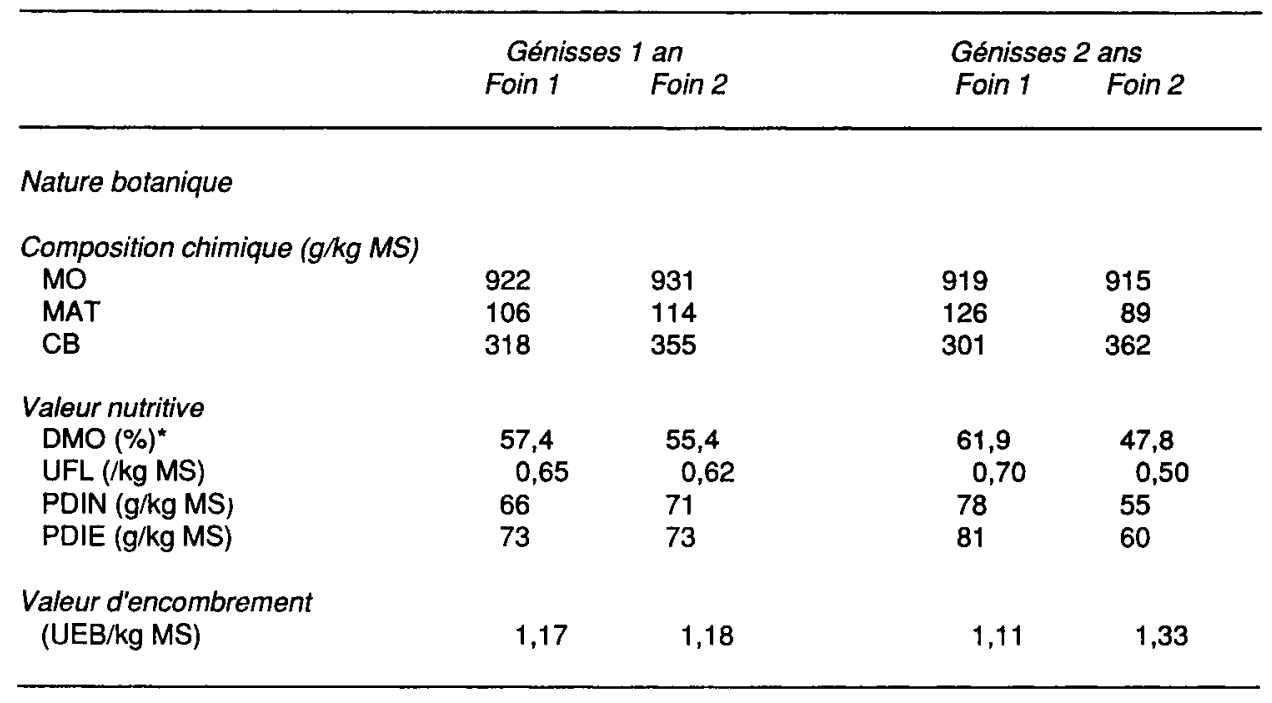

* Obtenue sur moutons.

calculées en effectuant une régression linéaire sur l'ensemble des poids vifs mesurés en période expérimentale afin d'en limiter les variations aléatoires.

Les températures minima et maxima de l'air dans les 2 stabulations ont été relevés quotidiennement, les thermomètres étant situés au niveau des animaux. Pendant la période expérimentale, la température moyenne de l'air a été plus faible en stabulation libre $\left(2,5^{\circ} \mathrm{C}\right)$ qu'en stabulation entravée $\left(12^{\circ} \mathrm{C}\right)$.

\section{Traitement des données}

Les 5 premières semaines de mesures, correspondant aux 3 semaines de pré-expérience sur le foin commun et aux 2 semaines d'adaptation après le changement de foin ont été éliminées de l'analyse pour conserver finalement 6 semaines.

Les résultats sur les animaux alimentés individuellement ont été traités, année par année, par analyse de variance selon un modèle tenant compte de la race, de la qualité du foin et du poids vif. Les variables analysées ont été, par animal, les quantités de foin ingérées et les quantités ingérées en pourcentage du poids vif (Ql/100 kg de poids vif). Les faibles quantités de tourteau de soja distribuées n'ont pas été prises en compte, les taux de substitution du concentré au fourrage etant probablement nuls dans ces conditions. La capacité d'ingestion (CI) individuelle observée des génisses, exprimée en UEB, a été calculée en multipliant les quantités de fourrages ingérées par leur valeur d'encombrement. Afin de regrouper les 2 classes d'âge, une analyse de variance a été effectuée selon le modèle suivant :

$$
\log (C l)=a \times \log (\text { poids })+\operatorname{race}
$$

en éliminant les génisses Salers car nous ne disposions pas de génisses Salers de 2 ans.

L'effet du mode de stabulation sur les quantités ingérées a été étudié sur la même période expérimentale de 6 semaines, en comparant les 
moyennes des valeurs individuelles des génisses de l'étable entravée recevant le même foin que les groupes de génisses élevées en stabulation libre.

\section{RÉSULTATS}

\section{Effet de la race}

Les quantités de foin ingérées ont été supérieures $(P<0,01)$ chez les Pie Noires et les Montbéliardes comparée aux Tarines et aux Salers (tableau III), essentiellement en raison des écarts de poids vif (fig 1). Celui-ci explique ainsi $58 \%$ des variations des quantités ingérées pour une même classe d'âge et $77 \%$ pour les 2 classes d'âge réunies. D'après les coefficients de régression, les quantités ingérées augmentent de $12 \mathrm{~g}$ de MS/ $\mathrm{kg}$ de poids vif pour les génisses de 1 an et de $15 \mathrm{~g}$ de $\mathrm{MS} / \mathrm{kg}$ pour celles de 2 ans. Ainsi, en rapportant les quantités ingérées au poids vif (tableau III), on ne distingue plus que 2 groupes de génisses : celles de race lajtière d'une part (Pie Noire, Montbéliarde et Tarine) dont la capacité d'ingestion est importante $(1,91 \mathrm{~kg} \mathrm{MS} / 100 \mathrm{~kg}$ de poids vif) et les génisses Salers d'autre part $(1,56 \mathrm{~kg}$ de $M S / 100 \mathrm{~kg}$ de poids vif). Cependant, les génisses montbéliardes ont eu tendance, mais de manière non significative,

Tableau III. Quantités ingérées (en kg de MS/j) par les génisses en stabulation entravée.

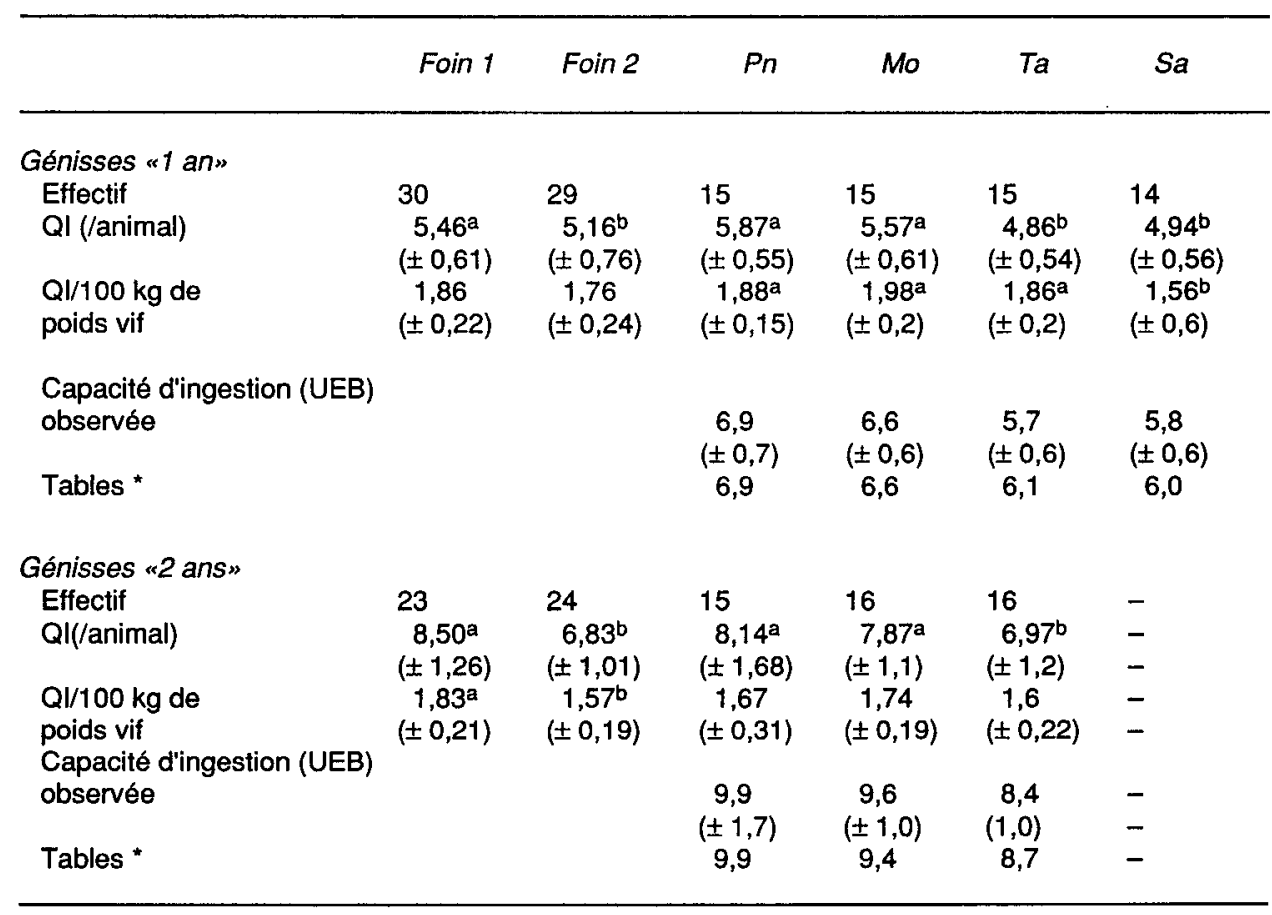

Intrafacteur, valeurs suivies de lettres differentes : probabilite $<5 \%$;

* calculée d'après Troccon et al (1988). 
à ingérer davantage $(+5 \%)$ pour un même poids vif que les Pie Noires et les Tarines.

La variabilité individuelle des quantités ingérées rapportées au poids vif est voisine entre les différentes races et les âges, les coefficients de variation variant de 9 à 15\%. Seules, les génisses Pie Noires de 2 ans présentent un coefficient de variation plus important $(21 \%)$, dû essentiellement à celles ayant reçu le meilleur foin (fig 1).

La capacité d'ingestion, exprimée en UEB, observée dans cet essai est peu différente de celle, théorique, calculée à partir du poids des animaux, et ce quelle que soit la race (tableau III). Elle est quasiment identique pour les Montbéliardes et légèrement plus faible pour les Tarines la première année $(-7 \%)$. Rapportée au poids vif des animaux (fig 2), elle est identique pour les génisses Pie Noires, Montbéliardes et Tarines, mais différente de celle des génisses Salers. En effectuant une transformation logarithmique sur les variables $\mathrm{Cl}$ et poids, le coefficient de la régression intrarace est de 0,80 et la part de variance expliquée par le poids est légèrement améliorée $(81 \%)$. Dans cette analyse, aucune différence significative n'apparaît entre les 3 races laitières.

\section{Effet de la qualité du foin}

La première année, l'écart de digestibilité de la matière organique ( $\mathrm{dMO}$ ) entre les 2 foins n'était que de 2 points (tableau II). La différence de quantités ingérées entre les 2 foins a alors été faible, de l'ordre de 0,3 $\mathrm{kg}$ (tableau III), et à peine significative $(P=$ $0,05)$. Par contre, la seconde année, les 2 foins étaient très différents, avec un écart de DMO de 14 points (tableau II), soit une variation de $0,2 \mathrm{UFL} / \mathrm{kg}$ de MS. Les quantités ingérées ont différé de $1,7 \mathrm{~kg}$ de $\mathrm{MS} / \mathrm{j}$ (tableau III, $P<0,01$ ).

Quelle que soit la race, et même lorsque la qualité des foins était très différente (année 2) les écarts de quantités ingérées ont été similaires (interaction race $x$ foin non significative les 2 années).

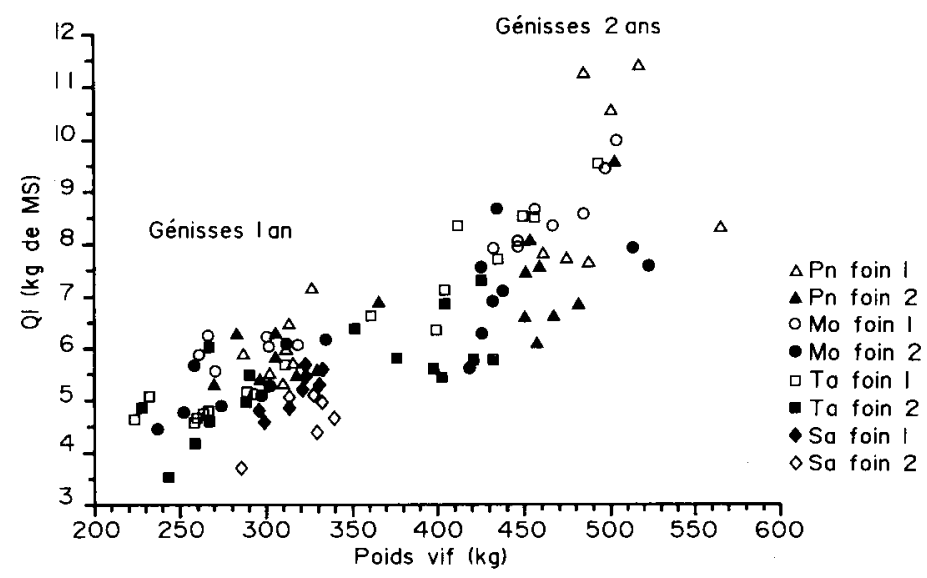

Fig 1. Quantité de foin ingéré (Ql en $\mathrm{kg}$ de MS/j) en fonction du poids vif (en $\mathrm{kg}$ ). 


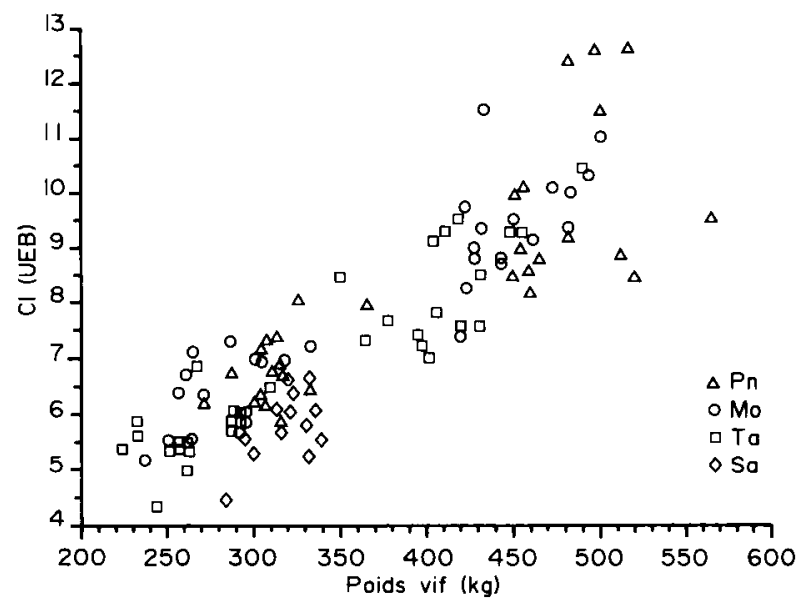

Fig 2. Évolution de la capacité d'ingestion ( $\mathrm{Cl}$ en UEB) en fonction du poids vif (en kg).

\section{Effet du type de stabulation}

Les 2 années, les génisses ont ingéré davantage en stabulation libre qu'en stabulation entravée (tableau IV). L'augmentation de consommation a été plus importante chez les Tarines $(+8$ et $+18 \%)$ et les Salers $(+9 \%)$ que chez les Pie Noires $(-3$ et $+9 \%$ ). La première année, les croissances des génisses ont été différentes entre la stabulation libre et entravée $(-100,+100$ et $+200 \mathrm{~g} / \mathrm{j}$ pour les Pie Noires, Tarines et Salers respectivement). La deuxième année, les croissances ont été semblables entre les types de logement. En stabulation entravée, l'augmentation de l'énergie ingérée a cependant toujours été supérieure à l'écart théorique des besoins de

Tableau IV. Influence du mode de stabulation sur les quantités ingérées (en kg de MS/)).

\begin{tabular}{|c|c|c|c|c|c|c|}
\hline & $P n$ & $\begin{array}{c}\text { Entrave } \\
\qquad \mathrm{Ta}\end{array}$ & Sa & Pn & $\begin{array}{l}\text { Libre } \\
\text { Ta }\end{array}$ & Sa \\
\hline \multicolumn{7}{|l|}{ Génisses «1 an» } \\
\hline $\begin{array}{l}\text { Effectif } \\
\text { QI(/animal) } \\
\text { Ql/100 kg de poids vif }\end{array}$ & $\begin{array}{l}7 \\
5,91 \\
1,89\end{array}$ & $\begin{array}{l}8 \\
4,96 \\
1,91\end{array}$ & $\begin{array}{l}8 \\
5,16 \\
1,63\end{array}$ & $\begin{array}{l}7 \\
5,71 \\
1,82\end{array}$ & $\begin{array}{l}8 \\
5,69 \\
2,06\end{array}$ & $\begin{array}{l}7 \\
5,57 \\
1,77\end{array}$ \\
\hline \multicolumn{7}{|l|}{ Génisses "2 ans» } \\
\hline $\begin{array}{l}\text { Effectif } \\
\text { QI//animal) } \\
\text { QI/100 kg de poids vif }\end{array}$ & $\begin{array}{l}7 \\
7,21 \\
1,54\end{array}$ & $\begin{array}{l}8 \\
6,12 \\
1,53\end{array}$ & $\begin{array}{l}- \\
- \\
-\end{array}$ & $\begin{array}{l}7 \\
7,65 \\
1,68\end{array}$ & $\begin{array}{l}8 \\
7,43 \\
1,82\end{array}$ & $\begin{array}{l}- \\
-\end{array}$ \\
\hline
\end{tabular}


croissance correspondant $3 \%$ pour les Pie Noires et Salers, $15 \%$ pour les Tarines).

\section{DISCUSSION}

Dans cet essai, la capacité d'ingestion, à même poids vif, des génisses laitières a été supérieure de $15 \%$ à celle des génisses Salers ce qui est un peu plus important que l'écart de $10 \%$ habituellement retenu (Agabriel et al, 1987a et 1987b; Troccon et al, 1988). Cet écart plus important pourrait résulter de l'âge légèrement plus faible des génisses Salers (-2 mois) par rapport aux génisses laitières ainsi que leur forte croissance pendant la période précédant l'essai (780 g/j). Les 3 génotypes laitiers ont la même capacité d'ingestion, ce qui est conforme aux recommandations de Troccon et al (1988) pour les génisses des races Pie Noire et Montbéliarde. De même, les quantités ingérées par des vaches adultes Montbéliardes et Pie Noires, de poids et de production laitières comparables, sont identiques (Coulon et al, 1985). Ces résultats permettent de classer les génisses Tarines dans le même groupe que les $\mathrm{Pie}$ Noires et les Montbéliardes pour la prévision de leur capacité d'ingestion.

Pour un même foin, les quantités ingérées dépendent en premier lieu du poids vif (Agabriel et al, 1987a; Taylor et al, 1986), mais la variation individuelle reste importante, en particulier pour les Pie Noires de 2 ans, sans qu'il y ait d'explications satisfaisantes, notamment sur le plan sanitaire, et elle est 2 fois plus élevée que celle observée par différents auteurs (Taylor et al, 1986; Dulphy et al, 1990). L'état d'engraissement, qui est un des facteurs importants de variation de la capacité d'ingestion (Agabriel et al, 1987b) et pourrait expliquer une partie de cette variabilité, n'a pas été mesuré dans cet essai.

La capacité d'ingestion augmente avec le poids élevé à la puissance 0,8 , ce qui est proche du coefficient de 0,75 utilisé dans le calcul des UE (Dulphy et al, 1987), et correspond aux valeurs indiquées par Forbes (1986) pour des animaux en croissance recevant des aliments riches en cellulose. Sur des races britanniques, ce coefficient varie de 0,7 à 0,8 (Taylor et al, 1986). Agabriel et al (1987a) avaient trouvé, pour des femelles Pie Noires, Limousines et Salers, une valeur légèrement supérieure $(0,9)$, mais pour une gamme d'âge plus étendue ( $1-8$ ans).

Dans cet essai, nous n'avons pas noté d'interaction entre les races et la qualité du foin. Cela conforte le principe de calcul des quantités ingérées à partir de la capacité d'ingestion exprimée en "unités d'encombrement" comme il est proposé en France (Dulphy et al, 1987).

Lorsque les génisses sont en stabulation libre, les quantités ingérées augmentent de l'ordre de 10-15\%, même si les écarts sont parfois plus importants. L'augmentation de l'énergie ingérée, pour une même croissance varie de 5 à $15 \%$, ce qui correspond à la valeur proposée par Jarrige et al (1978) pour l'accroissement des besoins énergétiques d'entretien entre les 2 types de logement. Cette différence de besoins peut résulter essentiellement de déplacements plus importants des animaux et de plus, dans notre étude, par des témpératures de l'air plus faibles en stabulation libre qu'en stabulation entravée (Forbes, 1986).

\section{CONCLUSION}

Pour un même foin et quelle que soit la race ou l'âge des animaux, le poids est le 
principal facteur de variation des quantités ingérées. Les différences de capacité d'ingestion entre génisses de types laitier et allaitant sont confirmées. Ces résultats ont montré que la prévision des quantités ingérées par des génisses Tarines peut être réalisée en utilisant les valeurs proposées pour les génisses de race laitière, sous réserve de connaître le poids des génisses et la valeur d'encombrement des foins.

La validation du système des "unités d'encombrement" sur cet ensemble de données est satisfaisante, mais il reste cependant des incertitudes sur les causes des fortes variations individuelles d'ingestion observées dans cette étude.

\section{RÉFÉRENCES}

Agabriel J, D'Hour P, M Petit P (1987a) Influence de l'âge et de la race sur la capacité d'ingestion des femelles bovines. Reprod Nutr Dév 27(18), 211-212

Agabriel J, Dulphy JP, Micol D (1987b) Utilisation des foins pour la croissance et l'engraissement des bovins. In : Les fourrages secs : récolte, traitement, utilisation (Demarquilly $\mathrm{C}$, ed) INRA, Paris, 283-318

Andrieu J, Demarquilly C (1987) Valeurs nutritives des fourrages : tables et prévisions. Bull Tech CRZV Theix, INRA 70, 61-73
Coulon JB, Garel JP, Hoden A, Journet M, Lienard $G$ (1985) Production laitière en zone de montagne : effet pluri-annuels du type de ration hivernale et du niveau de complémentation. Bull Tech CRZV Theix, INRA 61, 31-48

Dulphy JP, Faverdin P, Micol D, Bocquier F (1987) Révision du système des Unités d'Encombrement (UE). Bull Tech CRZV Theix, INRA 70, 35-48

Dulphy JP, Carle B, Demarquilly C (1990) Quantités ingérées et activités alimentaires comparées des ovins, bovins et caprins recevant des fourrages conservés avec ou sans aliment concentré. - I. Étude descriptive. Ann Zootech 39, 95-111

Forbes JM (1986) The voluntary food intake of farm animals. Butterworths. Londres, $206 p$

Jarrige R, Guéguen L, Vermorel M (1978) Entretien. In : Alimentation des Ruminants. INRA Publications, Versailles, 207-216

Oldenbroek JK, van Eldik P (1980) Differences in feed intake between Holstein Friesian, Dutch Red and White and Dutch Friesan cattle. Livest Prod Sci 7, 13-23

Taylor St CS, Moore AJ, Thiessen RB (1986) Voluntary food intake in relation to body weight among British breed of cattle. Anim Prod 42, 11-18

Troccon JL, Berge P, Agabriel J (1988) Alimentation des veaux et génisses d'élevage. In : Alimentation des bovins, ovins et caprins (Jarrige R, ed) INRA, Paris, 201-212 